\title{
Peak to Average Power Reduction for Low-Power OFDM Systems
}

\author{
Everest W. Huang and Gregory W. Wornell \\ Massachusetts Institute of Technology \\ Cambridge, MA 02139 \\ Email: \{everest, gww $\} @$ mit.edu
}

\begin{abstract}
For Orthogonal Frequency-Division Multiplexing (OFDM) systems, Peak to Average Power Ratio (PAPR) can be a major impediment to efficient transmission due to the need to use inefficient highly linear amplifiers. This paper presents an iterative algorithm to reduce the PAPR of a low-power OFDM system by $3 \mathrm{~dB}$ at a clipping probability of $10^{-2}$, and over $5 \mathrm{~dB}$ for $10^{-5}$ with asymptotically no loss in code rate for low signal to noise ratios (SNR). The reduced PAPR allows the system to use a more efficient form of linear amplifier, for an overall reduction in transmitter power consumption by over a factor of three at low SNR. In addition, the algorithm does not require any side information to be transmitted to the receiver to allow decoding.
\end{abstract}

\section{INTRODUCTION}

From a communications standpoint, the goal of the transmitter is to maximize the reliable data rate for a given transmit power, or equivalently, minimize the energy required per transmitted bit. An amplifier will saturate if the instantaneous output value is too high, however, so peak output power is also an important constraint. Using a multicarrier modulation scheme such as OFDM tends to produce output signals with a high PAPR. This negatively affects amplifier efficiency by requiring the the average signal power to be scaled to prevent saturation, lowering the SNR at the receiver. Because of their low efficiency, linear amplifiers can consume a significant fraction of the total transmitter power consumption.

We define an OFDM symbol as the bandlimited time series resulting from the inverse Fourier transform of $N$ complex values. Each value is a frequency bin, and is typically chosen from a constellation such as 16-QAM. The peak of an OFDM symbol is the largest instantaneous power of the bandlimited time signal, while the average is over the entire time signal. Thus $0 \mathrm{~dB}$ PAPR indicates a constant amplitude time signal.

Many strategies have been proposed to reduce the PAPR of an OFDM or other multicarrier signal, which either try to shape the signal while encoding the bits, or after the encoding has been performed. Performing the PAPR reduction while encoding has the potential of greatly decreasing the PAPR of the resulting OFDM time signal, but designing good codes that have low PAPRs is difficult without an excessive reduction in the achievable data rate. For example, Complementary Golay codes can limit the PAPR to only $3 \mathrm{~dB}$ [1], [2], but the code rate falls to zero rapidly as the length of the OFDM symbol increases. It should be possible to limit the PAPR to $\ln N$ for a length $N$ OFDM symbol without losing any appreciable code rate [3]. For $N=128$, this corresponds to a PAPR of no more than $16 \mathrm{~dB}$, but no known codes exist.
Altering the OFDM signal after encoding typically leads to more modest reductions in the PAPR. These algorithms can have relatively small losses in data rate and low complexity while retaining good error correcting performance. For example, clipping [4] or other nonlinear operations [5] can reduce the dynamic range of the time signal. At the expense of some code rate to transmit additional information to the receiver, it is also possible to cancel peaks [6], introduce offsets [7], or add dummy bits [8], [9] into selected frequency bins.

In this paper, we describe an algorithm to reduce the PAPR for OFDM systems, which in turn increases the efficiency of the transmitter power amplifier. This algorithm does not require side information to be sent to the receiver, and can be easily added to an existing system without seriously disturbing any coding already present. The phase synthesis algorithm achieves reductions in PAPR of $3 \mathrm{~dB}$ or more with no rate penalty at low SNR, making it a good choice for low power OFDM systems. In this case, low SNR and low power OFDM systems are characterized by each OFDM frequency bin having a low SNR as seen by the receiver.

\section{PAPR Distribution AND CLIPPING PROBABILITY FOR OFDM SYMBOLS}

We first look at the PAPR distribution for OFDM symbols to determine how often clipping occurs for a given PAPR threshold and how much rate must be used to reduce the PAPR below that threshold. To determine the distribution of PAPR values for an OFDM symbol, we begin with the discrete-time frequency samples which correspond to the values in each frequency bin and model the time series of an OFDM symbol as the bandlimited interpolation of samples of a circularlysymmetric Gaussian random function. As [10] shows,

$$
\operatorname{Pr}\left(\mathrm{PAPR}>\mathrm{PAPR}_{o}\right)=1-\left(1-e^{-\mathrm{PAPR}_{\mathrm{o}}}\right)^{N}
$$

is the Complimentary Cumulative Distribution Function (CCDF) of the PAPR of a discrete-time OFDM symbol of length $N$, and the CCDF of the bandlimited interpolation of these signals is closely modeled by the CCDF of a discretetime OFDM symbol of length $2 N$.

The left plot of Fig. 1 shows the CCDF for the PAPR of an OFDM symbol with $N=128$, corresponding to the number of frequency bins for an MC-OFDM UWB (ultrawideband) system [11]. We see that the PAPR will be greater than $6 \mathrm{~dB}$ with very high probability, but an OFDM symbol with a PAPR greater than $10 \mathrm{~dB}$ will occur less than $1 \%$ 

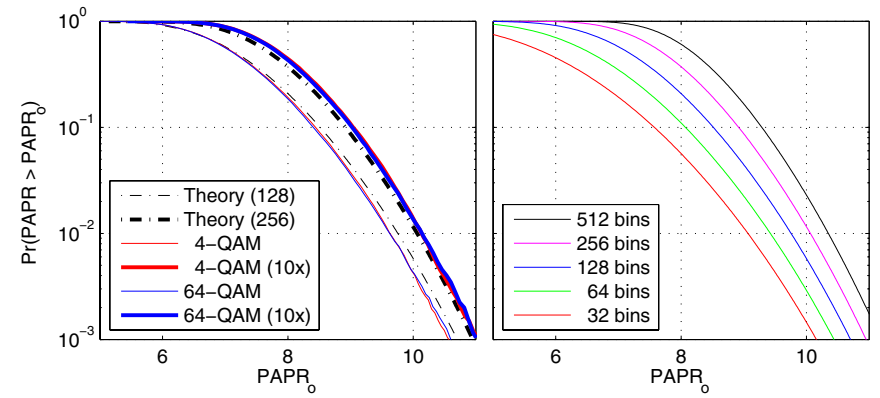

Fig. 1. Left shows PAPR CCDF for an $N=128$ OFDM symbol (thin lines) and its bandlimited interpolation (thick lines). Right plot has theoretical CCDFs given by (1) for $N=32,64,128,256$, and 512 (left to right).

of the time. The type of modulation used does not affect the peak distribution, as the plots for 4-QAM and 64-QAM follow the expected CCDF given by (1) closely, except at very low probabilities, which is an artifact of insufficient data points. Note that the PAPR of the actual OFDM symbol is limited to $N$, the number of frequency bins, but the Rayleigh distribution model used in (1) has a nonzero probability for all positive values. Also shown is the PAPR of the continuoustime signals (approximated here by $10 \times$ oversampling of the discrete-time signals, although in general $4 \times$ oversampling should be sufficient [12]), which have a distribution within about $0.5 \mathrm{~dB}$ of the expected CCDF from (1) for the PAPR of an OFDM symbol with twice as many frequency bins.

The right plot of Fig. 1 shows how the average PAPR increases as the length of the OFDM symbol increases, as well as how the probability density of PAPR values becomes more clustered around the mean value. As the number of frequency bins increases, the PAPR of a randomly selected OFDM symbol asymptotically approaches $\ln N$ with probability one [3]. One implication of this is that to reduce the PAPR to be less than $\ln N$ as $N \rightarrow \infty$ would require a significant drop in the code rate, while allowing a maximum PAPR of $\ln N$ requires a negligible loss in the code rate. Unfortunately, the specific combinations of frequency bin values that will create the OFDM symbols with high peaks are not easily avoided or predicted due to the nonlinear relationship between the frequency- and time-domain signals. Coding strategies do exist which will keep the PAPR no larger than $c \ln N$ for a constant $c$ [13], but for small values such as $N=128$ this corresponds to a PAPR limit of almost $16 \mathrm{~dB}$.

\section{EFFECT OF PAPR ON AMPLIFIER EFFICIENCY}

Because the amplitude and phase of each OFDM frequency bin are independently chosen, the corresponding time waveform will in general be very peaky. As a worst case example, the same constellation point is chosen in all the frequency bins. The time waveform will have a single peak at the first sample, and all others will be zero, resulting in a PAPR proportional to $N$. For a typical number of subcarriers, such a large PAPR can greatly reduce transmitter efficiency.
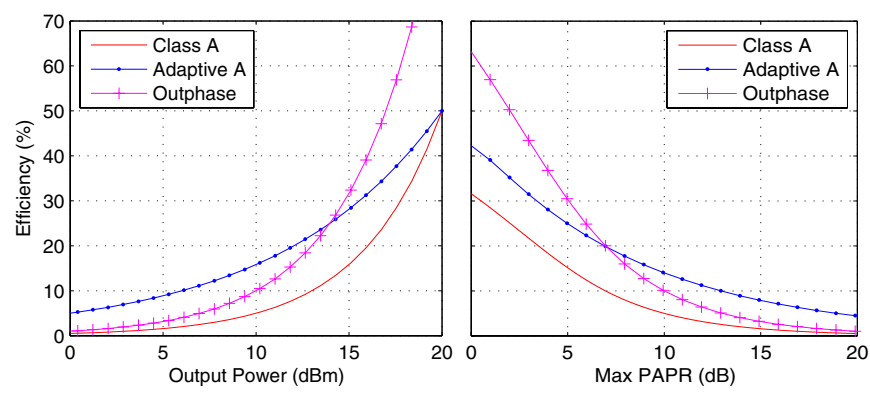

Fig. 2. Comparison of the instantaneous efficiency of various amplifier topologies as functions of the output power (left) and the average efficiency for a Rayleigh input distribution vs. maximum PAPR (right).

\section{A. Instantaneous Amplifier Efficiency Curves}

The instantaneous efficiency, defined as the ratio of the output power to the total power consumption, of a power amplifier depends strongly on both the input level as well as the type of amplifier used. Because of the high linearity demands of OFDM symbols, we restrict the analysis to linear amplifiers only. In addition to the common class A amplifier, we also consider more efficient linear amplifiers which adaptively bias the circuits [14] or combine the output of two constant amplitude amplifiers through outphasing [15].

The left plot of Fig. 2 shows the efficiency curves of these three types of amplifiers with respect to output power assuming ideal components and hard clipping at a maximum output of $20 \mathrm{dBm}(100 \mathrm{~mW})$. The class A amplifier has the worst efficiency, as expected, with the adaptive class A amplifier improving the efficiency somewhat, and the outphasing amplifier having the highest efficiency when within 5-6 dB of its maximum output power. Although the ideal efficiencies of these amplifiers can approach $100 \%$, typical maximum amplifier efficiencies are closer to $30 \%$ for both of the class A amplifiers, and 47\% for the outphasing amplifier [15].

\section{B. Average Efficiency with Rayleigh Inputs}

From the instantaneous efficiency curves, we can calculate the expected efficiency for a given clipping threshold for each amplifier [10]. The right plot of Fig. 2 plots the efficiency curves for these amplifiers for a Rayleigh magnitude distribution, which approximates the distribution of an OFDM symbol. Here, maximum PAPR denotes the largest PAPR that will not be clipped by the amplifier. Thus a maximum PAPR of $10 \mathrm{~dB}$ corresponds to an Input Back Off (IBO) of $10 \mathrm{~dB}$ for the average transmit power.

Although the class A amplifier can have a theoretical maximum efficiency of $50 \%$, the actual efficiency is often less than $10 \%$, and with a maximum PAPR of $10 \mathrm{~dB}$ corresponding to a $1 \%$ clipping level, the efficiency is only $5 \%$. The adaptive class A amplifier fares much better, but its efficiency is still less than $15 \%$ for a maximum PAPR of $10 \mathrm{~dB}$ or greater. If the PAPR could be limited to less than about $7 \mathrm{~dB}$, then the outphasing amplifier becomes the most efficient linear 
amplifier, approaching efficiency over $60 \%$. Even though the outphasing amplifier has the highest maximum efficiency, the Rayleigh distribution emphasizes the low magnitudes, which is where the amplifier efficiency is at its lowest. If nothing is done to reduce the large amplitude "tail" of the magnitude distribution, then the expected PAPR for a 64-QAM system with 128 frequency/time samples is about $16 \mathrm{~dB}$. None of the linear amplifiers are able to achieve an efficiency above $10 \%$ at this IBO, with the outphasing amplifier having an efficiency of only a few percent. Reducing the PAPR of the OFDM signal can greatly increase the efficiency of the amplifier by allowing the average power of the input to the amplifier to be increased without increasing the probability of clipping.

\section{Amplitude Synthesis FOR PAPR REDUCTION}

The proposed method to reduce the PAPR is inspired by a related problem in phased array design. A phased array alleviates the problems of having a high powered linear amplifier by summing the outputs of many amplifiers in free space. If the outputs of the small amplifiers are treated as samples of a continuous-time signal, then the resulting antenna pattern is given by the Fourier transform of the time samples [16]. The problem of trying to specify the amplitude and phases for each of the amplifiers to achieve a desired antenna pattern is very similar to trying to specify the amplitude and phases of the frequency bins to make the time signal a desired shape (in this case, a constant amplitude signal).

For each frequency bin in the OFDM symbol, $M$-QAM constellations are used, which encode the information in each bin with a magnitude and phase representing the appropriate constellation point. It is a known result that given only the frequency-domain magnitude of an unknown time signal which meets a mild set of conditions, it is possible to recover the frequency-domain phase of the unknown signal [17].

The PAPR synthesis algorithm only transmits information via the frequency magnitude of the OFDM symbol, and tries to "recover" the phase information that comes from a low PAPR time signal with the desired frequency magnitudes. At the transmitter, this operation comes at the cost of some of the data rate since information could have also been sent via the phase channel, as well as some computation complexity to synthesize the appropriate phases. At the receiver, the decoding only uses the magnitudes of the frequency bins and ignores the phases. The synthesis algorithm does not require information to be shared between the transmitter and receiver, and has a much higher complexity to encode rather than decode. This rate loss is prohibitive at high SNR, but at low SNR the rate loss vanishes because the phase channel has zero capacity, making this algorithm viable for low power applications.

\section{A. Capacity of Phase- and Magnitude-Only Channels}

Starting with a complex additive Gaussian channel,

$$
y=x+n,
$$

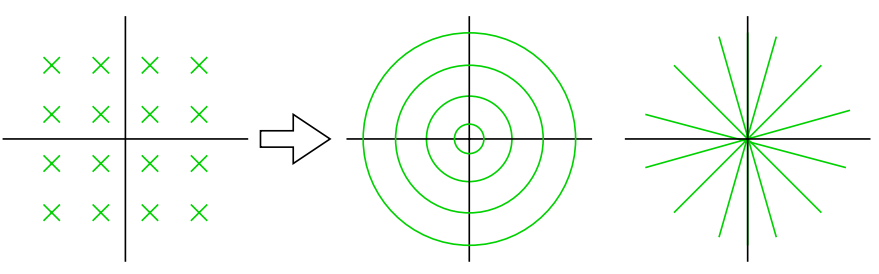

Fig. 3. A typical constellation for the complex (left), magnitude-only (middle), and phase-only (right) channels.

where $x$ is the input, $y$ is the output, and $n$ is additive white zero-mean Gaussian noise, with complex variance $2 \sigma^{2}$. The noise is uncorrelated with the input $x$. The complex channel can be viewed as two independent parallel real and imaginary channels, each with one-dimensional Gaussian noise with variance $\sigma^{2}$. The capacity given average input power $P$ is [18]

$$
C_{\text {real }}=C_{\text {imag }}=\frac{1}{2} \log _{2}\left(1+\frac{P}{\sigma^{2}}\right)
$$

in $\mathrm{b} / \mathrm{s} / \mathrm{Hz}$ where $P / \sigma^{2}$ is the SNR. The capacity of the complex channel $C_{\text {cplx }}$ is then the sum of the capacities for the real and imaginary channels.

Alternately, the complex channel can be decomposed into a magnitude-only and a phase-only channel. Fig. 3 shows what signal constellations might look like for these channels. The left plot is a 16-QAM constellation, which can be split up easily into two one-dimensional constellations, one each for the real and imaginary channels. The middle plot is what a constellation would look like for the magnitude-only channel, in which the phase is arbitrary. The result is concentric rings, with each ring representing a constellation "point." The right plot shows the constellation "points" for the phase-only channel. Because the magnitude is arbitrary, each ray represents a single constellation point, although given an average power constraint, the constellation points would be the intersection of these rays with a circle of radius $\sqrt{P}$.

The magnitude-only channel looks like

$$
y=|| x\left|e^{j \theta}+n\right|,
$$

where the magnitude $|x|$ encodes the information to be sent, and the angle $\theta$ is arbitrary. The noise $n$ is complex Gaussian as before, and the output $y$ is the magnitude of the noisy input signal. For this channel, the capacity in the high and low SNR regime is [19]

$$
C_{\mathrm{mag}} \approx \begin{cases}\frac{1}{2} C_{\mathrm{cplx}} & \frac{P}{\sigma^{2}} \gg 1 \\ C_{\mathrm{cplx}} & \frac{P}{\sigma^{2}} \ll 1 .\end{cases}
$$

At high SNR, the capacity of the magnitude-only channel is half that of the complex channel, which is the same as if information was only transmitted on the real channel. Thus restricting the input to only transmit information via the signal magnitude imposes a rate loss of one half. At very 
low SNR, however, the optimum distribution is Pulse-Position Modulation (PPM) [20], and there is no loss in capacity by using the magnitude-only channel. This is unlike the case of the complex channel, for which the Gaussian distribution is optimal for all SNR, and the real channel always has half the capacity of the complex channel. Numerical calculations of the capacity show that at $\mathrm{SNR}$ values near $0 \mathrm{~dB}$, the rate loss from ignoring phase is greater than one half, but not more than $60 \%$ when compared to the complex channel [21].

For an OFDM symbol, PPM could correspond to only transmitting energy on a single frequency bin, with the bin number carrying the signal information. The resulting time signal is then a complex sinusoid for the minimum possible PAPR. This leads to a great loss in code rate, however, with only seven bits transmitted per OFDM symbol with $N=128$.

The phase-only channel looks like

$$
y=L\left(\sqrt{P} e^{j \theta}+n\right),
$$

where the input $x$ is a constant magnitude signal with magnitude $\sqrt{P}$. The information is carried on the phase $\theta$, and $n$ is again complex Gaussian noise. The output $y$ is the phase of the input signal with additive noise. At high SNR, the capacity of the phase-only channel is [22]

$$
C_{\text {phase }} \approx \log _{2} \sqrt{\frac{4 \pi}{e} \frac{P}{\sigma^{2}}} \approx \frac{1}{2} \log _{2}\left(\frac{P}{\sigma^{2}}\right) .
$$

At high SNR, the capacity of the complex channel is

$$
C_{\mathrm{cplx}} \approx \log _{2}\left(\frac{P}{\sigma^{2}}\right),
$$

so the capacity of the phase-only channel is nearly half that of the complex channel. At low SNR, because the capacity of the magnitude-only channel is the same as that of the complex channel, the phase channel must have zero capacity.

Thus using only the magnitude of the channel to send information results in a rate loss of one half in the high SNR regime, and no rate loss in the low SNR regime. Additionally, since the frequency magnitude is usually scaled according to the channel conditions, allowing the magnitudes to be altered to reduce the PAPR would change the distribution of signal power in the frequency bins to an undesirable configuration for the wireless channel. On the other hand, allowing the phase to be altered has no effect on the power distribution between the frequency bins. The PAPR synthesis algorithm will therefore use the phase of the frequency bins as adjustable parameters to reduce the PAPR of the OFDM symbol.

\section{B. The Phase Synthesis Algorithm}

If the desired signal is a constant amplitude signal, with the frequency magnitudes specified by the information to be encoded, then the unknown frequency phase can be "recovered" to create the constant amplitude signal using the algorithm specified in [23]. The desired constant amplitude signal is

$$
X[f]=M[f] e^{j \theta[f]},
$$

where $M[f]$ is the magnitude of the frequency bins and $\theta[f]$ is the corresponding phase to be synthesized by the phase recovery algorithm.

The steps of the synthesis algorithm are then

1) Encode information bits in $M[f]$

2) Choose random phases for $\theta[f]$

3) Project $X[f]$ into time domain to get $x[t]$

a) Use IFFT to generate time domain signal

b) Clip signal to average power level to get $x^{\prime}[t]$

4) Project $x^{\prime}[t]$ into frequency domain and sample to get $X^{\prime}[f]$ with $M[f]$ and $\theta^{\prime}[f]$

a) use FFT to generate frequency domain signal $X^{\prime}[f]=M^{\prime}[f] e^{j \theta^{\prime}[f]}$

b) Replace magnitude $M^{\prime}[f]$ with $M[f]$, keep phase $\theta^{\prime}[f]$ as is

5) Go to Step 3 if maximum iterations not reached.

For each iteration, the frequency magnitude is forced to remain at its original value, but the phases are allowed to change. If a solution exists, the phase function should converge to whatever is necessary for the resulting frequency signal $X[f]$ to have a constant amplitude in the time domain.

The algorithm is similar to a POCS (projection onto convex sets) algorithm, which is an iterative procedure that converges to an element that is a member of two convex sets [24]. In this case, the two sets are amplitude-limited time signals, and frequency signals with a given frequency magnitude. Note that both the time and frequency signals of these sets are discrete, as the OFDM symbol only specifies the frequency magnitudes for a finite number of frequency bins.

The phase synthesis is not a POCS algorithm because the sets are not both convex. For a convex set, if $A$ and $B$ are both elements of the set, then for any $0 \leq \alpha \leq 1$ the weighted sum of the two elements $\alpha A+(1-\alpha) B$ is also in the set. The set of amplitude-limited time signals is convex, but the set of all signals with a specified frequency magnitude is not. For example, the sum of two signals with the same frequency magnitude but which are 180 degrees out of phase at every frequency is zero. A POCS algorithm is guaranteed to find a point in the intersection of the two sets, assuming it exists [24], but this is not guaranteed if the sets are not convex. It can be guaranteed, however, that the error signal never gets larger with each iteration, although it may never converge to zero even if a solution exists [25], [26].

In Step 3, the time signal is clipped to the average signal power. Since adjusting the phase of the frequency bins will not affect the total power of the signal, clipping to the average power will ideally limit the time signal to a constant amplitude, reducing the PAPR of the OFDM signal. To project into the other set, the signal is converted to the frequency domain, and then the magnitude is reset to the original specified frequency magnitude. While the frequency magnitude is constantly forced to the original values, the phases are allowed to change to whatever values will lower the PAPR. 
In general, for $N$ frequency samples, each time sample is a linear combination of the $N$ frequency magnitudes. The coefficients are in turn functions of the frequency phases as well as the sample number. Because $N$ equations require $N$ parameters to solve, all the phases are expected to be needed to make all the time sample magnitudes equal. Because the phases are relative, in general $N-1$ of the $N$ phases must be used to make the time samples constant magnitude.

The actual signal that is amplified is the bandlimited interpolation of these time samples, which is unlikely to be constant amplitude even though the time samples are, however. Additionally, although the error vector for the sampled signals is guaranteed to be nonincreasing, the deviation of the associated continuous-time signal has no such guarantee. Simulations show that the although the time samples monotonically converge to constant magnitude, the PAPR of the bandlimited interpolation will sometimes decrease and then increase.

It is therefore necessary to check the continuous-time PAPR after each iteration, keeping the current values only if they are better than any previous ones. As a result, the algorithm always runs for the maximum number of iterations and chooses the iteration that resulted in the lowest PAPR. The algorithm is not able to use the information about the continuous-time PAPR during the projections unless the original signal is oversampled, which is equivalent to expanding the bandwidth of the signal and incurs an undesired loss in rate.

For OFDM symbols with a cyclic prefix, the extension is straightforward. Since the cyclic prefix is a copy of part of the OFDM symbol, it has no effect on the PAPR when added to the OFDM symbol after the phase synthesis algorithm has been run. Alternatively, the cyclic prefix could be added before the phase synthesis algorithm, providing more degrees of freedom for PAPR reduction, but at the cost of more computation.

\section{Simulation Results}

In order to determine the performance of the synthesis algorithm, simulations were run over 460,000 randomly-generated 128 bin OFDM samples using 64-QAM constellations. The synthesis algorithms was able to reduce the PAPR by around $3 \mathrm{~dB}$ at a symbol error rate of $10^{-2}$, as shown in Fig. 4. The resulting PAPR at a clipping probability of $10^{-2}$ after 100 iterations is near the $7 \mathrm{~dB}$ needed for the outphasing amplifier to be most efficient of the linear amplifiers being considered. For $M$ iterations, the synthesis algorithm requires $2 M$ FFT operations and $M$ clipping operations, so the computational complexity scales linearly with the number of iterations. The difference between 100 and 200 iterations is negligible, and only about $0.5 \mathrm{~dB}$ is lost by restricting the iterations to ten. Simulations with 512 subcarriers show over $3.3 \mathrm{~dB}$ improvement at a clipping probability of $10^{-2}$ and 10 iterations. The larger number of subcarriers require more iterations before there are no more gains, however, with a $0.3 \mathrm{~dB}$ improvement from 100 to 200 iterations.

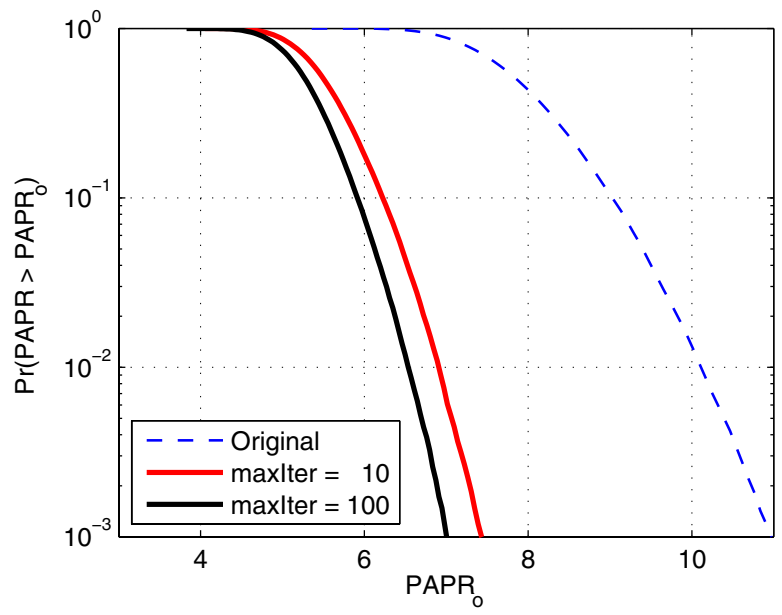

Fig. 4. CCDF of the PAPR for the phase synthesis algorithm for length 128 OFDM symbols using 64-QAM and $10 \times$ oversampling for bandlimited interpolation. The difference between 10 (solid right) and 100 (solid left) iterations is approximately $0.5 \mathrm{~dB}$

It should be noted again that these numbers are for the continuous-time PAPR. If we only considered the discrete-time PAPR, the synthesis algorithm reduces the PAPR to almost $0 \mathrm{~dB}$ even though the actual signal is not as constant magnitude as the discrete-time PAPR would suggest. This is a pitfall that is not always considered in other PAPR reduction schemes that have been proposed.

\section{A. Amplifier Efficiency Comparisons}

The ultimate goal is not to reduce the PAPR, but to improve the average efficiency of the amplifier. The average efficiencies of the OFDM symbols resulting from the simulations in the previous section were calculated, with each OFDM signal scaled such that the peak power of the signal is exactly at the saturation value of the amplifier to remove the effect of clipping. Because the transmitter has already calculated the peak of OFDM symbol for the synthesis algorithm, the computational impact of this operation is negligible.

Fig. 5 summarizes the average efficiency of the synthesis algorithm when combined with the three types of linear amplifiers and normalized to include the rate losses incurred by the magnitude-only output using the capacity curves from [21]. When the SNR is sufficiently low, the rate loss incurred by the synthesis algorithm approaches zero. The largest efficiency gain comes from using the synthesis algorithm with the outphasing amplifier. If the outphasing amplifier is too large for the target application, the adaptive class A amplifier is still able to achieve significant gains in efficiency when coupled with the phase synthesis algorithm. Even if it is not possible to change the amplifier from a class A (e.g., preexisting system design), using the phase synthesis algorithm can nearly double the amplifier efficiency. At higher SNR values, however, some of this efficiency gain is lost because many more symbols 


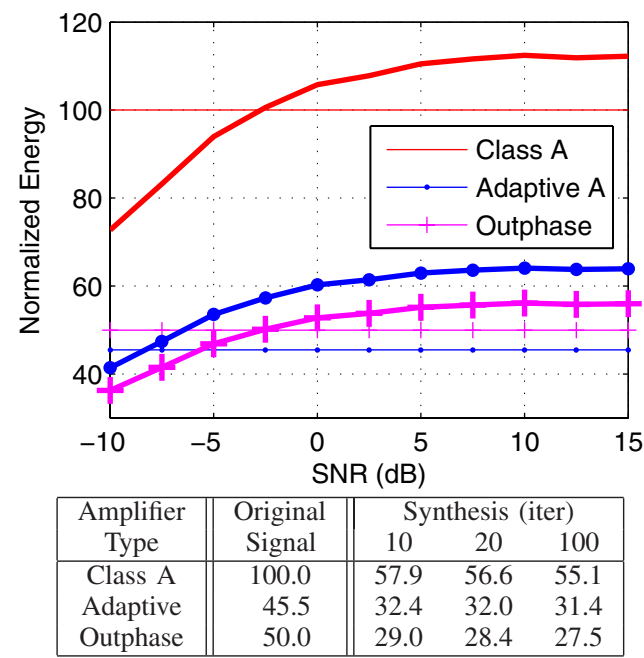

Fig. 5. Average energy per bit for three amplifier classes accounting for rate losses. Thick lines represent result of synthesis algorithm, and thin lines represent amplifier alone. Table shows average energy in the low SNR regime.

must be transmitted to achieve the same data rate as without the algorithm. As Fig. 5 shows, when the SNR is higher than $-2.5 \mathrm{~dB}$, the rate loss penalty makes the synthesis algorithm output unfavorable compared to the original signal.

For the proposed MC-OFDM UWB system in [11], the average transmitter output power is $-10.3 \mathrm{dBm}(93 \mu \mathrm{W})$, with a target SNR of around $4 \mathrm{~dB}$. Although this is above the break-even point of the synthesis algorithm, the instantaneous bandwidth of the OFDM symbols are only $7 \%$ of the total allocated bandwidth. Using the entire bandwidth shifts the overall SNR to $-7.5 \mathrm{~dB}$, where the rate loss from the synthesis algorithm is much smaller. From Fig. 2, we see that for a class A amplifier, the efficiency is about $1 \%$ for OFDM signals with $N=128$ frequency bins and no clipping or PAPR control. Thus to transmit $93 \mu \mathrm{W}$ requires almost $10 \mathrm{~mW}$ of power. With the synthesis algorithm and an outphasing amplifier, only about $2.5 \mathrm{~mW}$ of power is required. While this is less than $10 \%$ of the total power budget for the transmitter in [11], a slightly higher transmit power (e.g. $0 \mathrm{dBm}$, or $1 \mathrm{~mW}$ ) would quickly cause the amplifier to dominate the total power consumption.

\section{Vi. CONCLUSiOnS}

At low SNR, the synthesis algorithm is able to significantly reduce the PAPR with little or no rate loss, making it suitable for low power systems. Although the algorithm alone is able to increase the overall efficiency regardless of the amplifier used, the ability to optimize the type of amplifier to the PAPR algorithm can lead to noticeably larger performance gains.

\section{ACKNOWLEDGMENTS}

The authors would like to thank Don Boroson for many fruitful discussions.

\section{REFERENCES}

[1] B. M. Popovic̀, "Synthesis of Power Efficient Multitone Signals with Flat Amplitude Spectrum," IEEE Trans. Commun., vol. 39, no. 7, pp. 1031-1033, July 1991.

[2] J. A. Davis and J. Jedwab, "Peak-to-Mean Power Control in OFDM, Golay Complementary Sequences, and Reed-Muller Codes," IEEE Trans. Inform. Theory, vol. 45, no. 7, pp. 2397-2417, Nov. 1999.

[3] M. Sharif and B. Hassibi, "On Multicarrier Signals Where the PMEPR of a Random Codeword is Asymptotically $\log n$," IEEE Trans. Inform. Theory, vol. 50, no. 5, pp. 895-903, May 2004.

[4] X. Li and J. Leonard J. Cimini, "Effects of clipping and filtering on the performance of OFDM," in Proc. IEEE VTC (Spring), May 1997, pp. $1634-1638$

[5] X. Huang, J. Lu, J. Chuang, and J. Zheng, "Companding transform for the reduction of peak-to-average power ratio of OFDM signals," in Proc. IEEE VTC (Spring), May 2001, pp. 835-839.

[6] D. Farnese, A. Leva, G. Paltenghi, and A. Spalvieri, "Pulse Superposition: A Technique for Peak-to-Average Power Ratio Reduction in OFDM Modulation," in Proc. IEEE ICC, vol. 3, Apr. 2002, pp. 1682-1685.

[7] D. L. Jones, "Peak Power Reduction in OFDM and DMT via Active Channel Modification," in Proc. Asilomar Conf. Signals, Systems, and Computers, vol. 2, Oct. 1999, pp. 1076-1079.

[8] A. Gatherer and M. Polley, "Controlling Clipping Probability in DMT Transmissions," in Proc. Asilomar Conf. Signals, Systems, \& Computers, Nov. 1997, pp. $578-584$.

[9] J. Tellado and J. Cioffi, "Efficient Algorithms for Reducing PAR in Multicarrier Systems," in Proc. IEEE ISIT, Aug. 1998, p. 191.

[10] E. W. Huang, "Circuit-aware system design techniques for wireless communication," Ph.D. dissertation, MIT, Feb. 2006.

[11] A. Batra et al., Multi-band OFDM Physical Layer Proposal for IEEE 802.15 Task Group 3a, IEEE P802.15-03/268r3-TG3a, Mar. 2004.

[12] M. Sharif, M. Gharavi-Alkhansari, and B. H. Khalaj, "New Results on the Peak Power of OFDM Signals Based on Oversampling," in Proc. IEEE ICC, vol. 2, Apr. 2002, pp. 866-871.

[13] M. Sharif and B. Hassibi, "A determinisitic algorithm that achieves the PMEPR of $c \log n$ for multicarrier signals," in Proc. IEEE ICASSP, Apr. 2003, pp. 540-543.

[14] A. D. Pham, "Biasing techniques for linear power amplifiers," Master's thesis, MIT, 2002.

[15] _ _ "Outphase Power Amplifiers in OFDM Systems," Ph.D. dissertation, MIT, Sept. 2005.

[16] N. Amitay, V. Galindo, and C. P. Wu, Theory and Analysis of Phased Array Antennas. New York: Wiley-Interscience, 1972.

[17] J. Thomas F. Quatieri and A. V. Oppenheim, "Iterative Techniques for Minimum Phase Signal Reconstruction from Phase or Magnitude," IEEE Trans. Acoust., Speech, Signal Processing, vol. ASSP-29, no. 6, pp. 1187-1193, Dec. 1981

[18] T. M. Cover and J. A. Thomas, Elements of Information Theory. New York: John Wiley \& Sons, Inc., 1991.

[19] I. Jacobs, "Limits on the Power and Spectral Efficiency of Direct Detection Systems with Optical Amplifiers," in Proc. Asilomar Conf. Signals, Systems, \& Computers, vol. 1, Nov. 2001, pp. 8-12.

[20] M. J. E. Golay, "Note on the theoretical efficiency of information reception with PPM," Proc. IRE, vol. 37, p. 1031, Sept. 1949.

[21] M. Katz and S. Shamai, "On the Capacity-Achieving Distribution of the Discrete-Time Noncoherent and Partially Coherent AWGN Channels," IEEE Trans. Inform. Theory, vol. 50, no. 10, pp. 2257-2270, Oct. 2004.

[22] R. E. Blahut, Principles and Practice of Information Theory. Reading, MA: Addison Wesley, 1987.

[23] M. H. Hayes, J. S. Lim, and A. V. Oppenheim, "Signal Reconstruction from Phase or Magnitude," IEEE Trans. Acoust., Speech, Signal Processing, vol. ASSP-28, no. 6, pp. 672-680, Dec. 1980.

[24] P. L. Combettes, "The Foundations of Set Theoretic Estimation," Proc. IEEE, vol. 81, no. 2, pp. 182-208, Feb. 1993.

[25] O. M. Bucci, G. Mazzaralla, and G. Panariello, "Reconfigurable Arrays by Phase-Only Control," IEEE Trans. Antennas Propagat., vol. 39, no. 7, pp. 919-925, July 1991.

[26] H. Stark, W. C. Catino, and J. L. LoCicero, "Design of phase gratings by generalized projections," J. Opt. Soc. Am. A, vol. 8, no. 3, pp. 566-571, Mar. 1991. 